\title{
Passive Smoking During the Periconceptional Period and Risk for Neural Tube Defects in Offspring - Five Counties, Shanxi Province, China, 2010-2016
}

\author{
Yali Zhang ${ }^{1,2}$; Jufen Liu' ${ }^{1,2}$; Le Zhang ${ }^{1,2}$; Lei Jin ${ }^{1,2} ;$ Nicholas D E Greene ${ }^{3}$; Zhiwen Li ${ }^{1,2,2} ;$ Aiguo Ren ${ }^{1,2}$
}

\section{Summary}

What is already known about this topic?

Passive smoking during pregnancy, which is prevalent in China, has been reported to be associated with an increased risk for neural tube defects (NTDs) in five counties of Shanxi Province in northern China.

What is added by this report?

After 4 years since the implementation of the World Health Organization (WHO) Framework Convention on Tobacco Control (FCTC) in China, $59.4 \%$ of mothers of NTDs cases and $29.4 \%$ of mothers of healthy controls reported passive smoking during the periconceptional period. The association between periconceptional passive smoking and an increased risk for NTDs remains in the study population.

What are the implications for public health practice?

It's urgent to take measures to prevent passive smoking among pregnant women to minimize the harmful effects on offspring.

Neural tube defects (NTDs) are severe congenital malformations in the central nervous system, which include anencephaly, spina bifida, and encephalocele. Research has confirmed that folic acid supplementation during the periconceptional period has a protective effect (1-2). Nonetheless, some NTDs are not preventable by folic acid supplementation. Periconceptional exposure to passive smoking has been reported to increase the risk of NTDs (3-4). The adverse effects of maternal passive smoking as a potential risk factor for NTDs is getting more attention (5). China is the largest producer and consumer of tobacco products in the world, with more than 300 million smokers and 740 million nonsmokers exposed to second-hand smoke (6). Since the World Health Organization (WHO) Framework Convention on Tobacco Control (FCTC) officially took effect in China in 2006, China has taken strong measures to ban smoking in public places. This study aimed to investigate the effect of maternal passive smoking exposure on the risk of NTDs in offspring in five counties within Shanxi Province of China after 4 years of FCTC implementation. The results showed that there was an association between periconceptional passive smoking and an increased risk for NTDs. Measures need to be taken to prevent passive smoking among pregnant women to minimize the harmful effects on offspring.

Data were analyzed from an ongoing case-control study based on population-based birth-defect surveillance system that covered five counties (Xiyang, Pingding, Taigu, Shouyang, and Zezhou) in Shanxi Province. The surveillance system monitors major external structural birth defects that were diagnosed prenatally or neonatally. When one case with any major external structural birth defect including NTDs was identified, one healthy newborn infant of the same sex in the same region and with the closest date of conception to the case was selected as the healthy control. Information was collected through in-person interviews by trained healthcare workers before discharge from the hospital within 10 days after delivery, using a structured questionnaire including demographics, gravidity history, lifestyle behaviors, maternal active smoking, and passive smoking. For the current study, we included the subjects investigated from 2010 to 2016 . The study excluded 12 women (1.5\%) who reported active smoking and 27 women $(3.4 \%)$ whose active or passive smoking status were missing. This study defined passive smoking as exposure to secondhand smoke at least once per week and at least one cigarette each time on average in their environment (such as at home, in the workspace or other public places) from 1 month before to 2 months after pregnancy. The study was approved by the Institutional Review Board of Peking University. Written informed consent was provided by all participants. 
Logistic regression was used to evaluate the association between maternal passive smoking and risk for NTDs by crude odds ratio (OR) including 95\% confidence intervals (95\% CIs) and adjusted OR including $95 \%$ CIs after adjusting for potential confounders. The confounding variables included maternal age, pre-pregnancy body mass index (BMI), education, fever or flu in early pregnancy, nausea and vomiting during pregnancy (NVP), periconceptional folic acid use, and history of birth defect-affected pregnancy. All analyses were performed using SPSS package (Version 24.0. IBM Corp., Armonk, NY, USA).

A total of 224 NTD cases and 523 controls were included in analyses from 2010 to 2016. The case group comprised 78 cases of anencephaly, 121 of spina bifida, and 25 of encephalocele. Among 224 NTDs cases, 16 cases $(7.1 \%)$ had other malformations, such as orofacial clefts or gastroschisis. Compared with healthy control mothers, case mothers had lower education levels, higher gravidity, higher frequency of history of pregnancy affected by birth defects, higher prevalence of contracting fever or flu or having NVP, and lower rates of periconceptional folic acid use. In addition, case mothers were more likely than control mothers to be $\geq 30$ years of age and to be overweight (Pre-pregnancy BMI $\geq 25 \mathrm{~kg} / \mathrm{m}^{2}$, Table 1 ).

Overall, 59.4\% (133/224) of case mothers reported exposure to passive smoking during the periconceptional period, compared with $29.4 \%$ (154/523) of control mothers. In 2013, we started collecting data on places where passive smoking occurs (e.g., at home, work, or in other public places). There were 87 women who reported the information, of whom $62.1 \%$ were exposed to smoke at home. The crude OR of NTDs for passive smoking exposure was 3.502 (95\% CI: 2.527-4.853). After adjusting for maternal age, pre-pregnancy BMI, education, fever or flu in early pregnancy, NVP, periconceptional folic acid use, and history of birth defect-affected pregnancy, there was a still strong association, with the adjusted OR being 3.227 (95\% CI: 2.213-4.704). Positive dose-response patterns between exposure frequency and risk of NTDs were found, and the adjusted ORs were 1.878 (95\% CI: 1.115-3.163), 2.584 (95\% CI: $1.348-4.954)$, and 6.114 (95\% CI: 3.673-10.178) for exposure frequency of $1-3,4-6$, and $>6$ times per week respectively $\left(P_{\text {for trend }}<0.001\right.$, Table 2). Analysis of sub-types of NTDs separately showed that anencephaly, spina bifida, and encephalocele were also significantly associated with maternal passive smoking exposure after adjusting for potential confounders (Table 3).

\section{DISCUSSION}

This study indicated an association between maternal passive smoking during the periconceptional period and an increased risk for both total NTDs and subtypes in offspring of the nonsmoking women, with a significant dose-response relationship. A previous study reported that periconceptional exposure on partner smoking might increase the risk of NTDs in the offspring in same regions from 2002 to 2007 $(\mathrm{OR}=1.7$; 95\% CI: 1.3-2.4) (3). Another populationbased case-control study consisting of 175 cases and 221 controls among Mexican American women found a similar association between secondhand smoke exposure and NTDs (OR=2.6; 95\% CI: 1.6-4.0) (7). Recently, a meta-analysis reported that maternal passive smoking conferred an approximately two-fold risk increase of NTDs (adjusted OR $=1.898 ; 95 \%$ CI: 1.557-2.313) (5). These findings were consistent with our study, but the effect was weaker than what we observed in our study (adjusted $\mathrm{OR}=3.227$; 95\% CI: 2.213-4.704). This study clarified that higher exposure levels of maternal passive smoking were associated with improved risk for NTDs in offspring, with evidence of a dose-response pattern in nonsmoking women. Most notably, the risk for NTDs rapidly increased to a high level when the exposure frequency was $>6$ times per week (adjusted OR=6.114; 95\% CI: 3.673-10.178).

Maternal exposure to passive smoking during pregnancy increases the risk of adverse pregnancy outcomes (8). In China, female passive smoking is widespread and severe due to the high smoking rates among men, particularly in rural areas. A Chinese study reported the passive smoking exposure rate was $44.9 \%$ among 75,107 adults aged $\geq 40$ years (9). The passive smoking exposure proportions recorded in this study were $59.4 \%$ in the case group and $29.4 \%$ in the control group. The harmful constituents in cigarette smoke can pass through the placenta, including nicotine, carbon monoxide, and polycyclic aromatic hydrocarbons (10). However, most participants are not aware of the health dangers of passive smoking. The WHO FCTC was signed and put into effect in 2006, but unfortunately, tobacco control policies in China are poor and a large gap exists from the FCTC requirements (6). This study suggests that control measures should be taken to prevent tobacco smoke pollution from affecting pregnant women, particularly 
TABLE 1. Demographic and lifestyle characteristics of NTDs cases and controls in five counties, Shanxi Province, China, 2010-2016.

\begin{tabular}{|c|c|c|c|c|c|}
\hline \multirow{2}{*}{ Characteristic } & \multicolumn{2}{|c|}{ NTDs cases $(n=224)$ * } & \multicolumn{2}{|c|}{ Controls $(n=523)^{*}$} & \multirow{2}{*}{$P$} \\
\hline & Number & $\%$ & Number & $\%$ & \\
\hline Maternal age (years) & & & & & 0.005 \\
\hline$<25$ & 82 & 37.6 & 253 & 49.3 & \\
\hline $25-29$ & 71 & 32.6 & 161 & 31.4 & \\
\hline $30-34$ & 43 & 19.7 & 71 & 13.8 & \\
\hline$\geq 35$ & 22 & 10.1 & 28 & 5.5 & \\
\hline Prepregnancy BMI $\left(\mathrm{kg} / \mathrm{m}^{2}\right)$ & & & & & $<0.001$ \\
\hline$<18.5$ & 21 & 9.7 & 50 & 9.7 & \\
\hline $18.5-24.9$ & 124 & 57.4 & 364 & 70.8 & \\
\hline$\geq 25$ & 71 & 32.9 & 100 & 19.5 & \\
\hline Occupation & & & & & 0.094 \\
\hline Farmer & 169 & 75.4 & 362 & 69.2 & \\
\hline Others & 55 & 24.6 & 161 & 30.8 & \\
\hline Education & & & & & $<0.001$ \\
\hline Primary school or lower & 18 & 8.1 & 24 & 4.6 & \\
\hline Junior high school & 150 & 67.3 & 284 & 54.6 & \\
\hline Senior high school & 37 & 16.6 & 107 & 20.6 & \\
\hline College or higher & 18 & 8.1 & 105 & 20.2 & \\
\hline Gravidity & & & & & 0.004 \\
\hline 1 & 88 & 39.6 & 247 & 47.8 & \\
\hline 2 & 75 & 33.8 & 186 & 36.0 & \\
\hline$\geq 3$ & 59 & 26.6 & 84 & 16.2 & \\
\hline Parity & & & & & 0.323 \\
\hline Primiparas & 120 & 57.1 & 305 & 61.1 & \\
\hline Multiparas & 90 & 42.9 & 194 & 38.9 & \\
\hline History of birth defect-affected pregnancy & & & & & $<0.001$ \\
\hline Yes & 12 & 5.5 & 5 & 1.0 & \\
\hline No & 208 & 94.5 & 507 & 99.0 & \\
\hline Fever or flu in early pregnancy & & & & & $<0.001$ \\
\hline Yes & 84 & 38.7 & 84 & 16.6 & \\
\hline No & 133 & 61.3 & 421 & 83.4 & \\
\hline Nausea and vomiting of pregnancy & & & & & 0.001 \\
\hline Yes & 53 & 24.5 & 73 & 14.2 & \\
\hline No & 163 & 75.5 & 442 & 85.8 & \\
\hline Maternal alcohol consumption & & & & & 0.078 \\
\hline Yes & 23 & 10.3 & 34 & 6.5 & \\
\hline No & 201 & 89.7 & 487 & 93.5 & \\
\hline Periconception folic acid use & & & & & 0.043 \\
\hline Yes & 129 & 58.1 & 337 & 65.9 & \\
\hline No & 93 & 41.9 & 174 & 34.1 & \\
\hline
\end{tabular}

* Values may not sum to the total numbers because of missing information for some subjects.

Abbreviations: NTDs=neural tube defects; BMI=body mass index. 
TABLE 2. Association between periconceptional passive smoking and risk for fetal NTDs in five counties, Shanxi Province, China, 2010-2016.

\begin{tabular}{ccccc}
\hline Passive smoking exposure & Case & Control & Crude OR (95\% Cl) & Adjusted OR* (95\% Cl) \\
\hline No & 91 & 369 & 1.0 & 1.0 \\
Yes (any exposure) & 133 & 154 & $3.502(2.527-4.853)$ & $3.227(2.213-4.704)$ \\
1-3 times/week & 37 & 75 & $2.000(1.268-3.155)$ & $1.878(1.115-3.163)$ \\
4-6 times/week & 24 & 37 & $2.630(1.499-4.617)$ & $2.584(1.348-4.954)$ \\
$>$ > times/week & 72 & 42 & $6.951(4.458-10.840)$ & $6.114(3.673-10.178)$ \\
\hline
\end{tabular}

* Adjusted for maternal age, pregnancy BMI, education, fever or flu in early pregnancy, nausea and vomiting during pregnancy (NVP), periconception folic acid use and history of birth defect-affected pregnancy; $P_{\text {trend }}<0.001$.

Abbreviations: NTDs=neural tube defects; $\mathrm{OR}=$ odds ratio; $\mathrm{Cl}=$ confidence interval; $\mathrm{BMI}=$ body mass index.

TABLE 3. Association between periconceptional passive smoking and risk for fetal NTDs subtypes in five counties, Shanxi Province, China, 2010-2016.

\begin{tabular}{ccccc}
\hline Groups & Number & Number of passive smoking exposure & Crude OR (95\% Cl) & Adjusted OR* (95\% Cl) \\
\hline Control & 523 & 154 & 1.0 & 1.0 \\
Anencephaly & 78 & 42 & $2.795(1.724-4.532)$ & $2.497(1.433-4.352)$ \\
Spina bifida & 121 & 78 & $4.346(2.864-6.596)$ & $3.907(2.404-6.348)$ \\
Encephaloceles & 25 & 13 & $2.596(1.158-5.817)$ & $2.540(1.043-6.188)$ \\
\hline
\end{tabular}

${ }^{*}$ Adjusted for maternal age, prepregnancy BMI, education, fever or flu in early pregnancy, nausea and vomiting during pregnancy (NVP), periconception folic acid use and history of birth defect-affected pregnancy.

Abbreviations: NTDs=neural tube defects; $\mathrm{OR}=$ odds ratio; $\mathrm{Cl}=$ confidence interval; $\mathrm{BMI}=$ body mass index.

exposure at home, to minimize the harmful effects of passive smoking exposure in offspring.

This study had several strengths. It was based on a population-based birth-defect surveillance system with a high prevalence of NTDs and a high participation rate. The case and control participants were from the same source population. The passive smoking exposure level was graded, which helped us to assess the association between different exposure levels and the risk of NTDs. Only nonsmoking mothers were selected in the current study, and the effect of passive smoking exposure was unconfounded by maternal active smoking.

This study was also subject to some limitations. Maternal passive smoking was reported mainly by pregnant women and reporting or recall bias may have potential impacts on the results due to the case-control design. It is worth further investigating with deeper passive smoking exposure assessments, especially through biochemical markers such as nicotine levels in maternal urine or umbilical cord blood. Studies that also considered potential gene-environment interaction may be necessary to understand the role of passive smoking exposure in NTDs etiology.

Acknowledgements: The participants of the study, and the staff of the five hospitals from Xiyang, Pingding, Taigu, Shouyang, and Zezhou counties.

Funding: National Natural Science Foundation of
China (No. 81973056); Medical Research Council (Grant Ref: MR/T003847/1) from UK.

doi: $10.46234 / \mathrm{ccdcw} 2021.193$

\# Corresponding author: Zhiwen Li, lizw@bjmu.edu.cn.

\begin{abstract}
${ }^{1}$ Institute of Reproductive and Child Health/Key Laboratory of Reproductive Health, National Health Commission of the People's Republic of China, Peking University, Beijing, China; ${ }^{2}$ Department of Epidemiology and Biostatistics, School of Public Health, Peking University, Beijing, China; ${ }^{3}$ UCL Great Ormond Street Institute of Child Health, University College London, London, UK.
\end{abstract}

Submitted: August 10, 2021; Accepted: September 02, 2021

\section{REFERENCES}

1. Berry RJ, Li Z, Erickson JD, Li S, Moore CA, Wang H, et al. Prevention of neural-tube defects with folic acid in China. China-U. S. Collaborative Project for Neural Tube Defect Prevention. N Engl J Med 1999; 341(20): 1485-90. http://www.ncbi.nlm.nih.gov/pubmed/ 10559448.

2. Copp AJ, Stanier P, Greene NDE. Neural tube defects: recent advances, unsolved questions, and controversies. Lancet Neurol 2013; 12(8): 799-810. http://www.ncbi.nlm.nih.gov/pubmed/23790957.

3. Li ZW, Zhang L, Ye RW, Liu JM, Pei LJ, Zheng XJ, et al. Partner cigarette smoking and risk of neural tube defects among infants of nonsmoking women in northern China. Tob Control 2013; 22(6): 401-5. https://tobaccocontrol.bmj.com/content/tobaccocontrol/22/6/401.full. pdf.

4. Li ZW, Liu JM, Ren AG, Zhang L, Guo ZY, Li Z. Maternal passive smoking and the risk of neural tube defects: a case-control study in Shanxi province, China. Chin J Epidemiol 2008;29(5):417-20. http:// qikan.cqvip.com/Qikan/Article/Detail?id=27146274. (In Chinese).

5. Meng X, Sun YX, Duan WH, Jia CQ. Meta-analysis of the association of maternal smoking and passive smoking during pregnancy with neural 
tube defects. Int J Gynecol Obstet 2018;140(1):18 - 25. http://dx. doi.org/10.1002/ijgo.12334.

6. Chinese Center for Disease Control and Prevention. Global adult tobacco survey (GATS): China 2010 country report. Beijing, China: China Three Gorges Press. 2011. (In Chinese)

7. Suarez L, Felkner M, Brender JD, Canfield M, Hendricks K. Maternal exposures to cigarette smoke, alcohol, and street drugs and neural tube defect occurrence in offspring. Matern Child Health J 2008;12(3):394 - 401. http://dx.doi.org/10.1007/s10995-007-0251-y.

8. Hoyt AT, Canfield MA, Romitti PA, Botto LD, Anderka MT, Krikov $\mathrm{SV}$, et al. Does maternal exposure to secondhand tobacco smoke during pregnancy increase the risk for preterm or small-for-gestational age birth? Matern Child Health J 2018;22(10):1418 - 29. http://dx.doi.org/ 10.1007/s10995-018-2522-1.

9. Cong S, Feng YJ, Bao HL, Wang N, Fan J, Wang BH, et al. Analysis on passive smoking exposure in adults aged 40 years and older in China, 2014. Chin J Epidemiol 2018;39(5):557 - 62. http://dx.doi.org/10. 3760/cma.j.issn.0254-6450.2018.05.004. (In Chinese).

10. Meeker JD, Missmer SA, Vitonis AF, Cramer DW, Hauser R. Risk of spontaneous abortion in women with childhood exposure to parental cigarette smoke. Am J Epidemiol 2007;166(5):571 - 5. http://dx. doi.org/10.1093/aje/kwm128. 